\title{
Retinal vascular permeability suppression by topical application of a novel VEGFR2/Src kinase inhibitor in mice and rabbits
}

\author{
Lea Scheppke, ${ }^{1}$ Edith Aguilar, ${ }^{1}$ Ray F. Gariano, ${ }^{1}$ Ruth Jacobson, ${ }^{1}$ John Hood, ${ }^{2}$ \\ John Doukas, ${ }^{2}$ Jon Cao, ${ }^{2}$ Glenn Noronha, ${ }^{2}$ Shiyin Yee, ${ }^{2}$ Sara Weis, ${ }^{3}$ \\ Michael B. Martin, ${ }^{2}$ Richard Soll, ${ }^{2}$ David A. Cheresh, ${ }^{3}$ and Martin Friedlander ${ }^{1}$ \\ 1Department of Cell Biology, The Scripps Research Institute, La Jolla, California, USA. \\ ${ }^{2}$ TargeGen Inc., San Diego, California, USA. ${ }^{3}$ Moores Cancer Center, UCSD, La Jolla, California, USA.
}

\begin{abstract}
Retinal and choroidal vascular diseases, with their associated abnormalities in vascular permeability, account for the majority of patients with vision loss in industrialized nations. VEGF is upregulated in ischemic retinopathies such as diabetes and is known to dramatically alter vascular permeability in a number of nonocular tissues via Src kinase-regulated signaling pathways. VEGF antagonists are currently in clinical use for treating the new blood vessels and retinal edema associated with neovascular eye diseases, but such therapies require repeated intraocular injections. We have found that vascular leakage following intravitreal administration of VEGF in mice was abolished by systemic or topical delivery of what we believe is a novel VEGFR2/Src kinase inhibitor; this was confirmed in rabbits. The relevance of Src inhibition to VEGF-associated alterations in vascular permeability was further substantiated by genetic studies in which VEGF injection or laser-induced vascular permeability failed to augment retinal vascular permeability in $\mathrm{Src}^{-/-}$and $\mathrm{Yes}^{-/-}$mice (Src and Yes are ubiquitously expressed Src kinase family members; $\mathrm{Src}^{-/-}$and $\mathrm{Yes}^{-/}$mice lacking expression of these kinases show no vascular leak in response to VEGF). These findings establish a role for Src kinase in VEGF-mediated retinal vascular permeability and establish a potentially safe and painless topically applied therapeutic option for treating vision loss due to neovascular-associated retinal edema.
\end{abstract}

\section{Introduction}

Abnormal retinal vascular permeability leading to edema in the area of the macula is the leading cause of vision loss in diseases such as diabetic retinopathy, exudative macular degeneration, retinal vascular occlusions, and inflammatory and neoplastic conditions $(1,2)$. Although a variety of disease processes may lead to increased vascular permeability through different mechanisms, the cytokine VEGF activates pathways of vascular leakage common to many. Increased vascular permeability in ischemic retinopathies and possibly also in exudative macular degeneration and uveitis, for example, correlates with VEGF levels (3-8). In fact, VEGF antagonists have been successfully used to reduce retinal/macular edema in neovascular eye diseases such as age-related macular degeneration with stabilization or even improvement of visual acuity in a subset of affected patients (9).

Recent studies have shown that VEGF-induced vascular leakage is mediated by cytoplasmic protein kinase members of the Src protooncogene family in brain, heart, and other tissues (10-13). Such vascular permeability is likely related to a loss of integrity in adherens junctions, which regulate cell-cell adhesion. Adherens junctions are complexes of cadherins, $\mathrm{Ca}^{2+}$-dependent transmembrane proteins, and catenins, cytoplasmic proteins that link the complex to the actin cytoskeleton. VEGF has been shown to activate the Src family of tyrosine kinases (SFKs), leading to tyrosine phosphorylation of adhe-

Nonstandard abbreviations used: EB, Evans blue; FAK, focal adhesion kinase; SFK, Src family of tyrosine kinase.

Conflict of interest: J. Hood, J. Doukas, J. Cao, G. Noronha, S. Yee, M.B. Martin and R. Soll were employees of TargeGen Inc. when the experiments reported in this manuscript were conducted. D.A. Cheresh and M. Friedlander were paid consultants (Scientific Advisory Board members) of TargeGen Inc.

Citation for this article: J. Clin. Invest. 118:2337-2346 (2008). doi:10.1172/JCI33361. sion junction components, including VE-cadherin and its associated proteins $\beta$-catenin and $\gamma$-catenin, important to endothelial cell adhesion (10, 13-16). The SFKs thought to be involved in VEGF-mediated vascular permeability include Src and Yes, which are expressed on all endothelial cells. Src preferentially associates with VEGFR2 (KDR in human, FLK-1 in mouse), while Yes associates preferentially with VEGFR1 (Flt-1) (17). Inhibitors of Src kinase activity prevent VEGF-induced vascular permeability, block angiogenesis, and reduce edema and functional loss associated with tumors and experimental cerebral vascular occlusion and myocardial infarction $(12,13)$. Cerebral and myocardial vessel permeability due to ischemia or to VEGF administration is markedly diminished in mice deficient in SFK family members $\operatorname{Src}$ and Yes $(12,13)$, as is metastatic invasion of cancer, a process that requires passage of malignant cells across vascular endothelia by diapedesis (18).

These findings led us to consider whether Src kinases participate in VEGF- and vasoocclusive-mediated vascular permeability in the retina. Our results support this hypothesis, and furthermore, we report the synthesis of a class of VEGFR/Src kinase antagonists that may be applied topically, accumulate at high levels in the retina, retain biological activity, and potently inhibit VEGF-mediated vascular permeability. Such topically applied inhibitors of Src kinase activity may prove valuable in the clinical management of diseases in which excessive retinal edema due to VEGF-mediated abnormalities in vascular permeability leads to loss of vision.

\section{Results}

VEGF-induced retinal vascular permeability. In order to evaluate VEGF-induced retinal leakage, mice were subjected to intravitreal administration of VEGF and monitored for extravasation of FITC- 

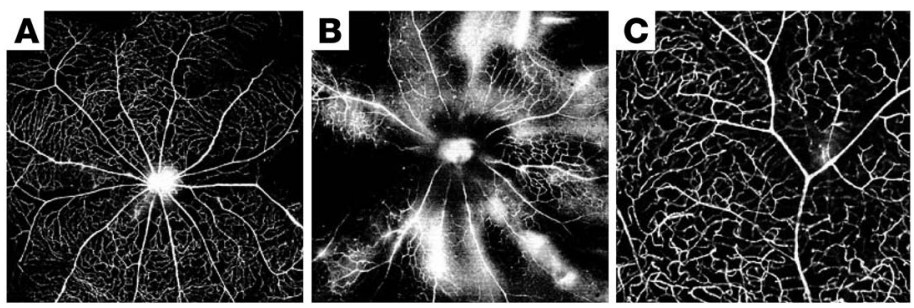

\section{D}

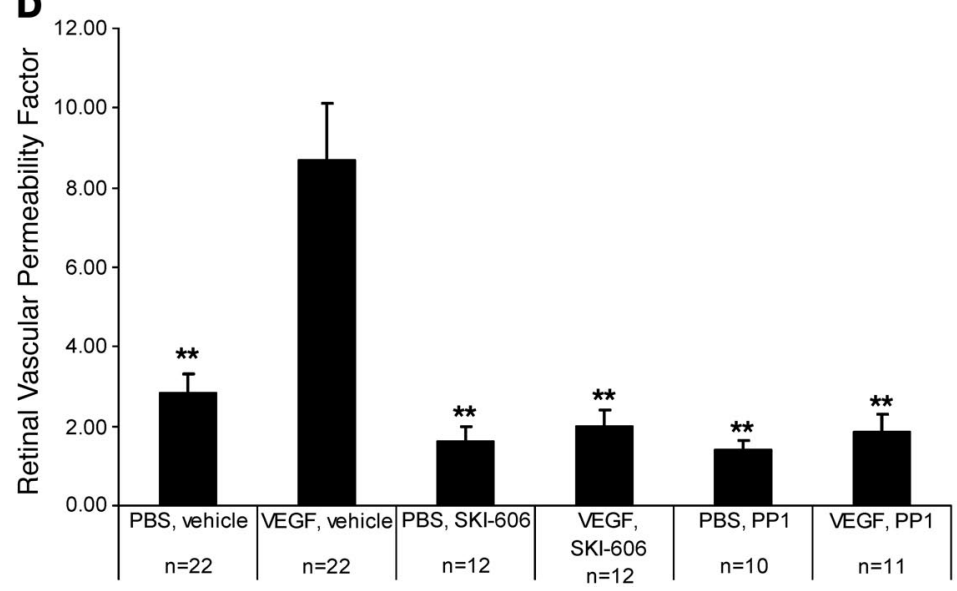

Figure 1

PP1 inhibits VEGF-induced retinal vascular permeability. (A-C) Fluorescein dextran-perfused retinal whole mounts from mice that received systemic vehicle and either intravitreal PBS (A) or VEGF (B) versus systemic PP1 and intravitreal VEGF (C). Shown is the absence of leakage in eyes given both VEGF and PP1 (C, higher magnification) compared with VEGF-treated eyes in the absence of an Src kinase inhibitor, which have areas of both focal and diffuse dextran extravasation (B). (D) Vascular permeability as measured by retinal EB dye accumulation, with and without drug treatments. Error bars indicate SEM. $n$ = eyes per group. EB leakage: intravitreal PBS and systemic vehicle, $2.81 \pm 0.51$ (SEM). VEGF/vehicle, $8.69 \pm 1.43$; PBS/SKI-606, $1.62 \pm 0.37$; VEGF/SKI-606, $1.98 \pm 0.43 ;$ PBS/PP1, $1.38 \pm 0.27 ;$ VEGF/PP1, $1.85+0.46$ Retinal vascular permeability factor is a measure of [concentration of EB dye in the retina]/[concentration of EB dye in the plasma $\times$ circulation time]. ${ }^{\star *} P<0.01$. Original magnification, $\times 4($ A, B $) ; \times 10($ C). dextran and FITC-albumin. Extravasation of these markers was evident (Figure 1, A and B) relative to control mice not treated with VEGF. Vascular leakage in VEGF-treated animals was indicated by a diffuse hyperfluorescent background and by patchy perivascular hyperfluorescence (Figure 1, A and B). Pretreatment with Src kinase inhibitors, either PP1 or SKI-606, diminished these angiographic features of VEGF-induced permeability (Figure 1C).

This vascular leak was quantified in a separate cohort of animals by measuring Evans blue (EB) dye extravasation from retinal vessels. Intraocular VEGF injection induced a 3-fold increase in retinal EB leakage compared with $\mathrm{PBS}$-injected eyes $(P=0.00038$; Figure 1D). In contrast, in animals pretreated with PP1 or SKI-606, VEGF-induced leakage was significantly inhibited $(P=0.0024$ and $P=0.0019$, respectively; Figure 1D). Overall, PP1 and SKI-606 prevented approximately $90 \%$ of VEGF-induced leak; Src kinase inhibition did not significantly alter retinal dye levels in the absence of VEGF stimulation (Figure 1D).

In vivo model of retinal hyperpermeability. At 3 days after laserinduced occlusion of retinal blood vessels, whole-mount angiography revealed marked retinal ischemia and blood-retinal barrier breakdown. Severe hypoperfusion and focal perivascular leakage of FITC-dextran or FITC-albumin appeared in all retinal quadrants, and diffuse background retinal fluorescence was brighter than in nonlasered eyes, despite the reduced perfusion (Figure 2, A and B). Inhibition of Src kinase activity by either PP1 or SKI-606 decreased but did not eliminate the angiographic evidence of vascular exudation (Figure 2C).

Retinal vascular permeability as indicated by EB dye accumulation increased approximately 5 -fold in eyes with laser vascular occlusion compared with untreated contralateral eyes $(P=0.004$; Figure $2 D)$. The effect of laser occlusion on dye accumulation persisted after administration of PP1 (2.5-fold increase; $P<0.009)$ but was $35 \%$ less than that measured in vehicle-injected animals $(P=0.25)$. Laser-induced retinal vascular leakage was not significantly elevat- ed after injection of SKI-606 $(P=0.07)$ but was $67 \%$ less than that measured in vehicle-treated animals $(P=0.015)$ (Figure 2D).

Vascular permeability in Src kinase mutant mice. Available Src kinase inhibitors interact with non-Src tyrosine kinases; for example, PP1 inhibits VEGFR2 and PDGF activity $(19,20)$, and SKI-606 is the most selective pharmacological inhibitor of Src kinase reported to date, as it selectively inhibits Src and Abl kinase but lacks growth factor receptor tyrosine kinase (21). Of note, Abl kinase is not known to play a role in VEGF-mediated edema in the retina, though it has been shown to be important for retinal neovascularization in a mouse model of retinopathy of prematurity (22), so the reduction of vascular permeability in response to SKI-606 is likely due to its inhibition of Src kinase. To further evaluate the role of SFKs in retinal vascular permeability (23), retinal vascular leak in $\mathrm{Src}^{-1-}$ and $\mathrm{Yes}^{-1-}$ mice was measured in response to intraocular VEGF injection. In both $\mathrm{Src}^{-/-}$and $\mathrm{Yes}^{-1-}$ mice, retinal whole-mount FITC dextran and albumin angiography appeared similar after intravitreal injection with either VEGF or PBS (Figure 3, A-C), suggesting that VEGF did not alter vascular permeability in eyes lacking these specific SFK members. Similarly, retinal EB content failed to increase after intravitreal VEGF administration in $\mathrm{Src}^{-/-}$and $\mathrm{Yes}^{-1-}$ mice $(P=0.95,0.98)$, though their respective wild-type littermates each responded to intravitreal VEGF with a significant rise in tracer levels $(P=0.015, P=0.019$, respectively; Figure $3 \mathrm{D})$.

While some extravasation of labeled dextran was detected following laser vasoocclusion in retinas of $\mathrm{Src}^{-/-}$and $\mathrm{Yes}^{-1-}$ mice, it was considerably less than that observed in lasered wild-type eyes (not shown). In wild-type littermates of both $\mathrm{Src}^{-/-}$and $\mathrm{Yes}^{-/-}$mice, laser treatment markedly augmented retinal vascular leakage $\left(\mathrm{Src}^{+/+}\right.$: 6.4-fold increase, $P=0.02 ;$ Yes $^{+/+}: 5.6$-fold increase, $P=0.00003$; Figure 4). In $\mathrm{Yes}^{-/-}$mice, laser treatment also significantly increased retinal EB accumulation compared with nonlasered eyes (2.8-fold increase, $P=0.00005)$. However, this effect of laser was $55 \%$ smaller than that observed in wild-type littermates (Figure 4). Src -knock- 

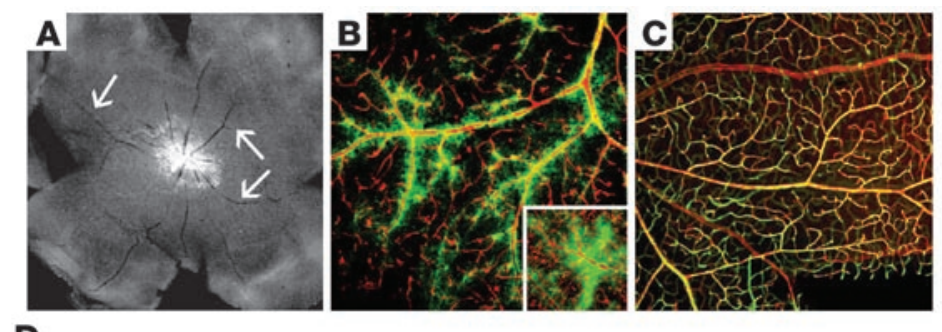

D

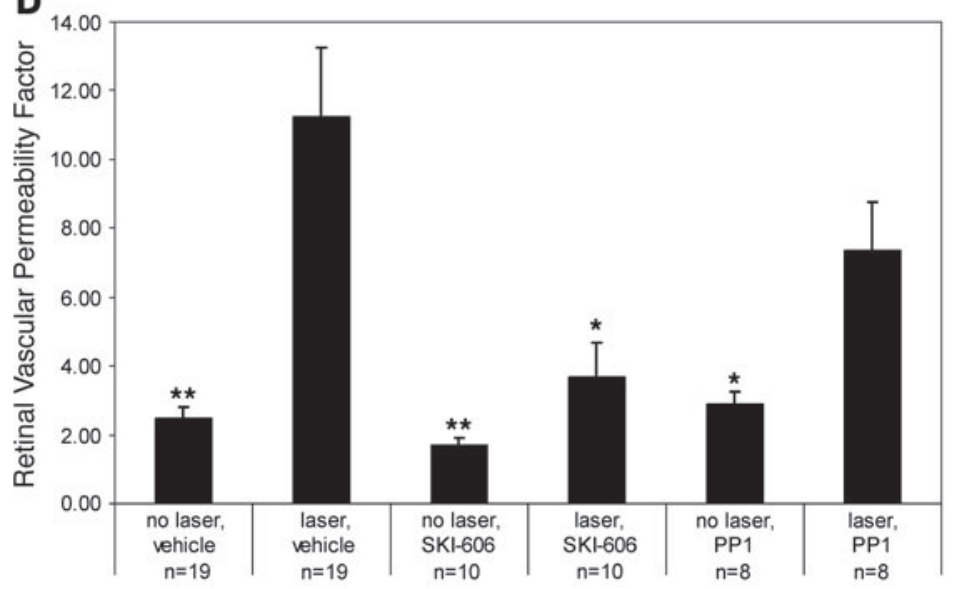

\section{Figure 2}

Src kinase antagonists reduce laser-induced retinal vascular permeability. (A-C) Whole-mount fluorescent dextran angiography 3 days after laser treatment. Ischemia is indicated by relatively hypoperfused major retinal vessels (arrows, A). Vascular leakage in laser-treated eyes is indicated by bright background fluorescence $(\mathbf{A})$ and by perivascular staining and leakage of tracer (B and inset, tracer green, vessels labeled red with isolectin). (C) SKI-606 treatment partly reduced angiographic evidence of vascular exudation in laser-treated eyes. (D) Retinal EB dye accumulation in control and laser-treated eyes; treatment with systemically administered PP1 or SKI-606 reduced laser-induced dye accumulation but did not alter leakage in nonlasered eyes. Error bars indicate SEM. No laser/systemic vehicle injection, $2.47 \pm 0.34$; laser/vehicle, $11.22 \pm 2.03$; no laser/SKI-606, $1.67 \pm 0.23$; laser/SKI-606, $3.69 \pm 1.01$; no laser/PP1, $2.86 \pm 0.38$; laser/PP1, $7.34 \pm 1.42$. Original magnification, $\times 4(\mathbf{A}) ; \times 10$ (inset, $\times 20)(\mathbf{B}, \mathbf{C}) .{ }^{*} P<0.05 ;{ }^{* \star} P<0.01$. out mice exerted a more pronounced suppression of laser-induced leakage: the increase in retinal EB dye accumulation after laser was not statistically significant (1.36-fold increase, $P=0.55 ; 76 \%$ less leakage than that induced in wild-type eyes).

A topically applied small molecule VEGFR2/Src kinase inbibitor permeates the cornea and is present and active in the retina. The VEGFR2/Src kinase inhibitor TG100572 evolved through a structure-based drug design approach to potent Src inhibitors of the benzotriazine class (24). TG100572 potently inhibits a number of tyrosine kinases responsible for mediating vascular leak and/or angiogenesis. $\mathrm{K}_{\mathrm{i}} \mathrm{s}$, which represent the binding affinity for TG100572 to the following targets are as follows: VEGFR2/KDR (6.8 nM), Src $(9.2 \mathrm{nM})$, Yes (0.6 nM), PDGFR $\beta$ (1.9 nM), FGFR1 (46 nM), and EphB4 (32 nM). In contrast, $\mathrm{IC}_{50}$ for TG100801 exceeds $300 \mathrm{nM}$ against these targets. TG100801 is a prodrug form of the active compound TG100572, which is cleaved into the active form in ocular tissues.

In order to confirm the capacity of TG100572 to inhibit its known molecular targets in vivo, the phosphorylation of focal adhesion kinase (FAK) on its Src phosphorylation site (tyrosine 861; FAK-Y861) was monitored in the choroid/sclera of mice undergoing developmental angiogenesis. TG100572 was injected i.p. into BALB/c mouse pups twice a day beginning on postnatal day 8 , a time of intense ocular angiogenesis as the deep vascular plexus emerges from the superficial plexus. Consistent with the involvement of VEGFR2 and Src kinase in this process, FAK-Y861 was heavily phosphorylated in retinal tissues (Figure 5). Specifically, TG100572 reduced FAK-Y861 phosphorylation in a dose-dependent manner, with more than $90 \%$ inhibition observed at the $5 \mathrm{mg} / \mathrm{kg}$ dose. $\beta$-actin was used as a loading control and was equal in all lanes. This confirms that the compound blocks signaling through this proangiogenic kinase pathway during ocular neovascularization.

Pharmacokinetics of topically applied inhibitors. Ocular tissue exposures to the prodrug TG100801 and its active compound TG100572 were evaluated in the mouse and rabbit following single topical bilateral instillation of 1.0\% TG100801 formulation currently under clinical evaluation (Figure 6). Topical administration of TG100801 provided substantial posterior ocular tissue exposure to TG100801 (Figure 6A) and TG100572 (Figure 6B) in the mouse (Supplemental Table 1; supplemental material available online with this article; doi:10.1172/JCI33361DS1). A single topical instillation of TG100801 in mice resulted in sustained ocular tissue concentrations of TG100801 and TG100572 with maximum posterior tissue concentrations observed at 0.5 to 1 hour after administration. Well-defined tissue concentration-time profiles (Figure 6, A and B) were observed for both TG100801 and TG100572, with maximum TG100572 tissue concentrations of $49 \pm 14 \mathrm{ng} / \mathrm{g}$ and $1230 \pm 373 \mathrm{ng} / \mathrm{g}$ for retinal and choroidal/scleral tissues, respectively. Conversion of TG100801 to the active TG100572 was observed in all ocular tissues. Following topical instillation of TG100801, plasma concentrations of TG100572 and TG100801 were below quantifiable levels $(<1 \mathrm{ng} / \mathrm{ml})$.

We recognize that the pharmacokinetics and permeability of topically applied compounds such as those used in this study may vary significantly depending on the geometry and size of the eye targeted. To assess the potential bioavailability and tissue distribution of such compounds in the human eye, we performed a series of experiments in which a larger animal eye that more closely approximated the human eye was used. The ocular tissue exposure to the prodrug TG100801 and its active compound TG100572 was evaluated in rabbit following topical bilateral instillation of $1.0 \%$ TG100801 (Figure 6, C and D). The rabbit eye approximates 70\% to $80 \%$ of the human eye in terms of axial length, diameter, corneal thickness, scleral thickness at the limbus, scleral thickness at the equator, and scleral surface area. Topical administration of TG100801 resulted in well-defined posterior ocular tissue concentration-time profiles for TG100801 (Figure 6C) and TG100572 (Figure 6D) in the rabbit with maximum posterior tissue concentrations observed at 2 to 4 hours after administration. Conversion 

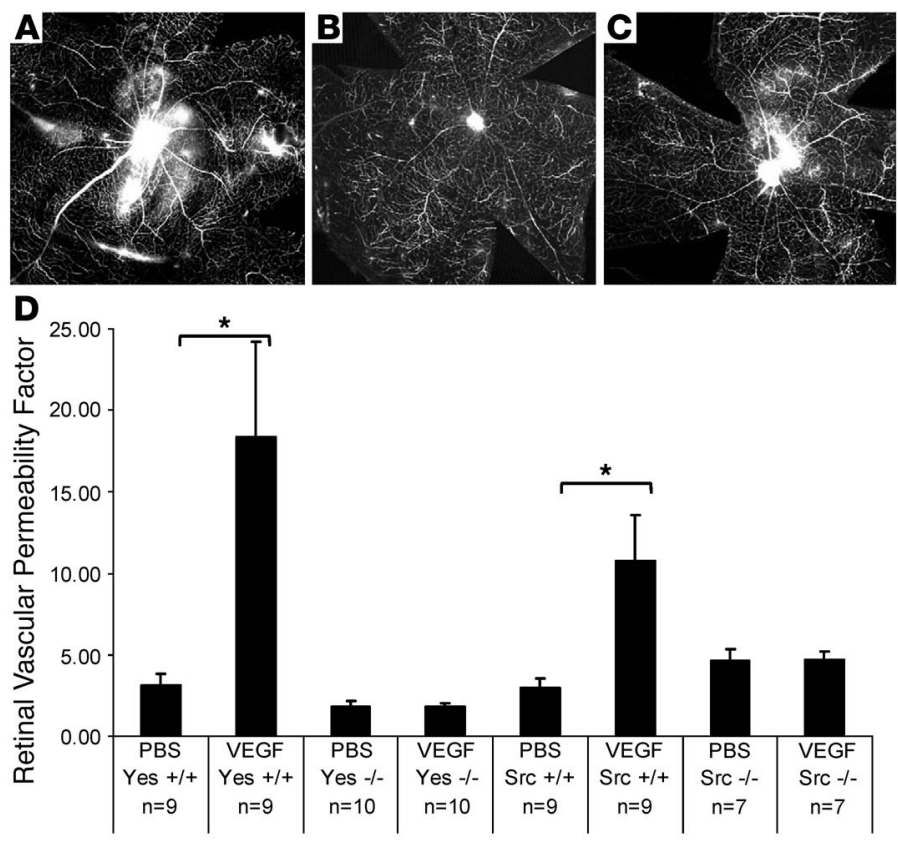

\section{Figure 3}

$\mathrm{Src}^{-/}$and $\mathrm{Yes}^{-/-}$mice are resistant to VEGF-induced retinal vascular permeability. (A-C) Whole-mount fluorescent-dextran angiograms in wild-type (A) and $\mathrm{SrC}^{-1-}$ (B and $\left.\mathbf{C}\right)$ littermates. Dextran leakage after VEGF injection was more marked in wild-type (A) than $\mathrm{SrC}^{-/}$ (C) eyes; vehicle injection was without effect (B). (D) In response to VEGF injection, EB accumulation was unaltered in $\mathrm{Src}^{-/}$and $\mathrm{Yes}^{-/}$retinas but was significantly augmented in Src and Yes wildtype littermates. Error bars indicate SEM. PBS/Yes ${ }^{+/+}, 3.07 \pm 0.77$; VEGF/Yes ${ }^{+/+}, 18.38 \pm 5.36, n=9$; PBS $/$ Yes $^{-/-}, 1.80 \pm 0.36$; VEGF/Yes ${ }^{-1-}, 1.79+0.24, n=10 ; \mathrm{PBS} / \mathrm{Src}^{-1-}, 2.98 \pm 0.53$; VEGF/ $\mathrm{Src}^{-/}, 10.78 \pm 2.83, n=9$; $\mathrm{PBS} / \mathrm{Src}^{-/}, 4.61 \pm 0.72$; VEGF/Src ${ }^{-/}$, $4.67 \pm 0.54, n=7$. Original magnification, $\times 4 .{ }^{*} P<0.05$. of TG100801 to the active TG100572 was observed in all ocular tissues, and plasma concentrations of TG100572 and TG100801 were below quantifiable levels $(<1 \mathrm{ng} / \mathrm{ml})$. The respective retinal concentrations of TG100801 and TG100572 were $410 \pm 195 \mathrm{ng} / \mathrm{g}$ and $51 \pm 27 \mathrm{ng} / \mathrm{g}$ in the rabbit (Supplemental Table 1), resulting in comparable concentrations of either prodrug TG100801 or active drug TG100572 between the mouse and the rabbit. In both species, the tissue concentration-time profiles display an apparent concentration gradient moving from high scleral concentrations to lower retinal concentrations, suggesting a transscleral delivery of the topically applied agent to the posterior tissues.

A topically applied small molecule VEGFR2/Src kinase inhibitor significantly reduced VEGF-mediated retinal vascular permeability. TG100801 or the active compound TG100572 was topically applied to the cornea 1-3 times during the course of the experiment. While a single administration of $0.7 \%$ TG100572 reduced retinal leakage by more than $50 \%$, the result was not statistically significant (Figure 7A). However, increasing the frequency of administration of the agent to 3 times per day resulted in more than $80 \%$ inhibition of VEGF-induced leak and a high degree of statistical significance (Figure 7B). To further optimize delivery to the back of the eye, thereby reducing the frequency of drug administration required for efficacy to one that is more relevant, a prodrug form of TG100572 was used. TG100801 was prepared as a $1.22 \%$ drop, topically administered once, and resulted in highly statistically significant and essentially complete inhibition of VEGF-induced retinal leak (Figure 7C).

In order to assess the efficacy of a VEGF antagonist, clinically used to inhibit retinal vascular permeability in the clinics, we decided to assess the effects of Macugen and Avastin in this model of retinal vascular permeability. Macugen, a recently approved intravitreally administered anti-VEGF therapy, was administered at $3.33 \mu \mathrm{g} /$ eye, which is the maximal dose feasible using the approved clinical concentration and formulation of the compound. Based on a calculation of the relative vitreous volumes between mice and humans and the quantities of Macugen injected, this resulted in an intravitreal concentration of drug approximately 4 times higher than has been shown to be efficacious clinically. Under these test conditions, Macugen inhibited blood-retinal-barrier breakdown by $54 \% \pm 19 \%$ $(P=0.05)$. Avastin, a recently approved i.v.-administered anti-VEGF antibody approved for colon cancer treatment, was administered systemically at $2 \mathrm{mg} / \mathrm{kg}$, a dose reported to be efficacious (9), resulting in a statistically significant inhibition of $84 \% \pm 13 \%(P<0.05)$.

Abnormal retinal vascular permeability is frequently associated with retinal neovascularization; thus, an antivascular permeability compound that is also antiangiogenic should have added therapeutic benefit. In order to evaluate the antiangiogenic activity of TG100572 hydrochloride, the mouse Matrigel model of angiogenesis was used. In this model, angiogenic blood vessels are induced to grow into a s.c.-implanted extracellular

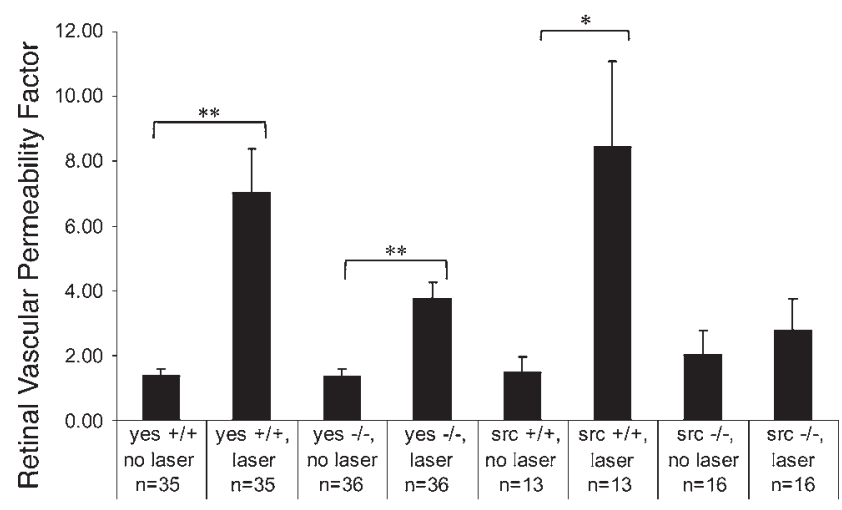

\section{Figure 4}

Laser vasoocclusion increases retinal vascular leakage more in wild-type $\left(\mathrm{Yes}^{+/+}, \mathrm{Src}^{+/+}\right)$than in Src kinase-deficient $\left(\mathrm{Src}^{-/}, \mathrm{Yes}^{-/-}\right)$ mice. $\left(Y_{e s}^{+/+} /\right.$no laser, $1.39 \pm 0.20 ; Y^{+/+/ l a s e r, ~} 7.04 \pm 1.33, n=35$;

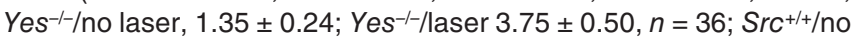
laser, $1.49 \pm 0.46$; $\mathrm{Src}^{+/+} / \mathrm{laser}, 8.44 \pm 2.66, n=13$; $\mathrm{Src}^{-/-/ n o ~ l a s e r}$, $2.04 \pm 0.74 ; \mathrm{SrC}^{-/}$/laser, $2.77 \pm 0.97, n=16$. Error bars indicate SEM. ${ }^{\star} P<0.05 ;{ }^{* \star} P<0.01$. 


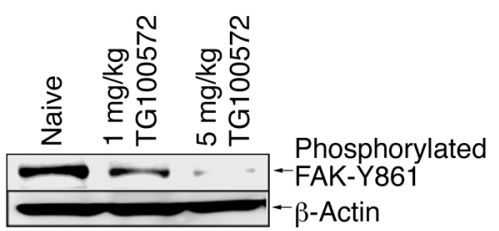

Figure 5

TG100572 inhibits FAK-Y861 phosphorylation during developmental angiogenesis. Choroid/sclera homogenate of BALB/c pups injected i.p. with TG100572 twice daily at the indicated doses.

matrix by either VEGF or FGF. Both of these growth factors have been shown to be potent proangiogenic factors, and a compound that inhibits redundant pathways of angiogenesis has the potential of being therapeutically advantageous. As shown in Figure 8, i.p. administration of TG100572 hydrochloride at $5 \mathrm{mg} / \mathrm{kg}$ once daily for 5 days revealed a statistically significant inhibition of both VEGF- and FGF-induced angiogenesis, reducing angiogenesis by $88 \% \pm 10 \%(P<0.00005)$ and by $100 \% \pm 24 \%$ $(P<0.002)$, respectively. Individual experimental data are provided in Supplemental Table 2.
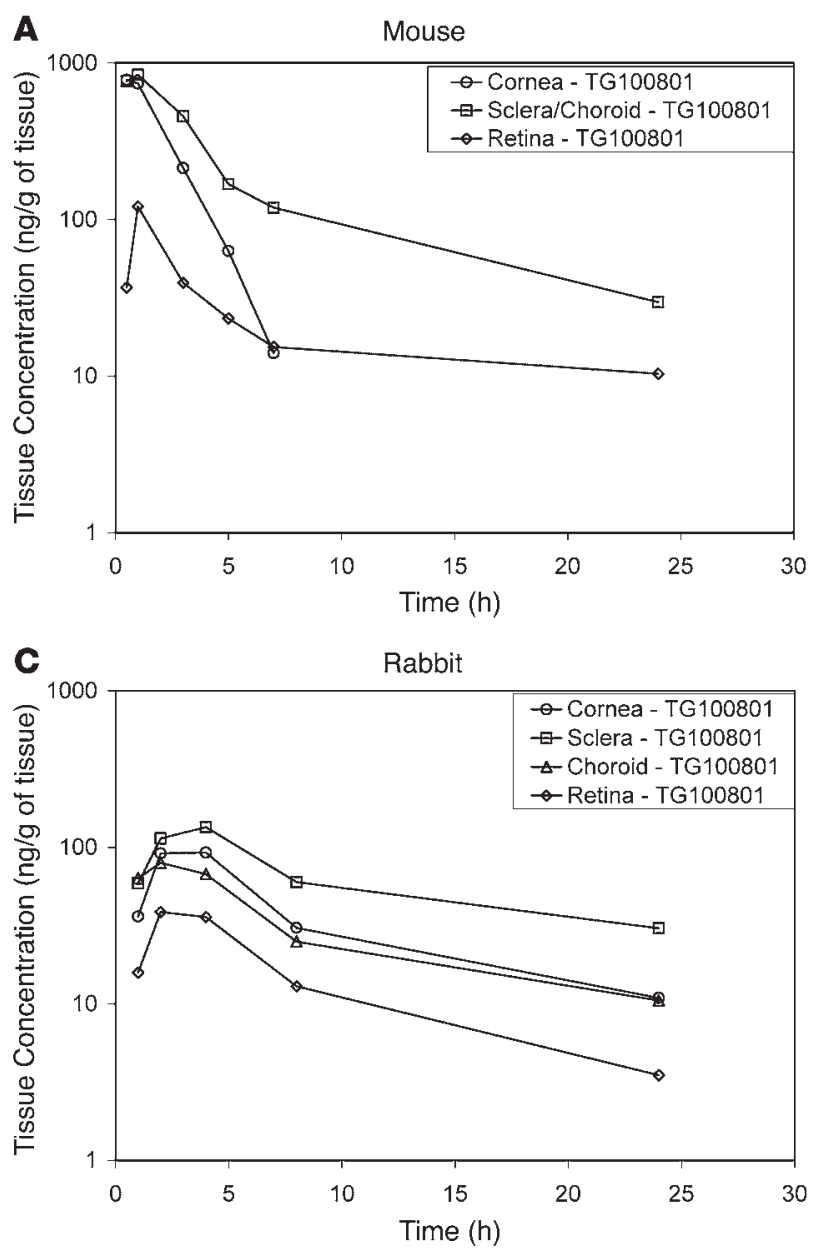

\section{Discussion}

Increased permeability of retinal and choroidal vessels is a common feature in diseases such as diabetes and age-related macular degeneration; fluid and exudative deposits in the central retina (macular edema) are the most common cause of visual loss in patients with diabetes (25). Presently, the only proven treatment for vascular leakage associated with ischemic retinopathies is laser photocoagulation, a modality that prevents vision loss in approximately half of eligible patients and improves vision infrequently $(25,26)$. Recent use of intravitreally injected VEGF antagonists demonstrates efficacy in stabilizing vision loss in $35 \%$ of patients with neovascular age-related macular degeneration, largely through the antipermeability effect of inhibiting VEGF activity $(9,27)$. Our demonstration that Src kinases participate in retinal vascular permeability and that VEGF- and ischemia-mediated vascular leakage are reduced by Src kinase inhibitors suggests what we believe is a novel therapeutic approach for these patients. While Src kinase activity appears critical for VEGF-induced permeability, it may be dispensable for mitogenic, neurotrophic, antiapoptotic, and other effects of VEGF $(28,29)$. Thus, inhibiting Src kinase may prevent VEGF-associated vascular permeability while preserving its angiogenic and cell-survival activities $(28,30)$.
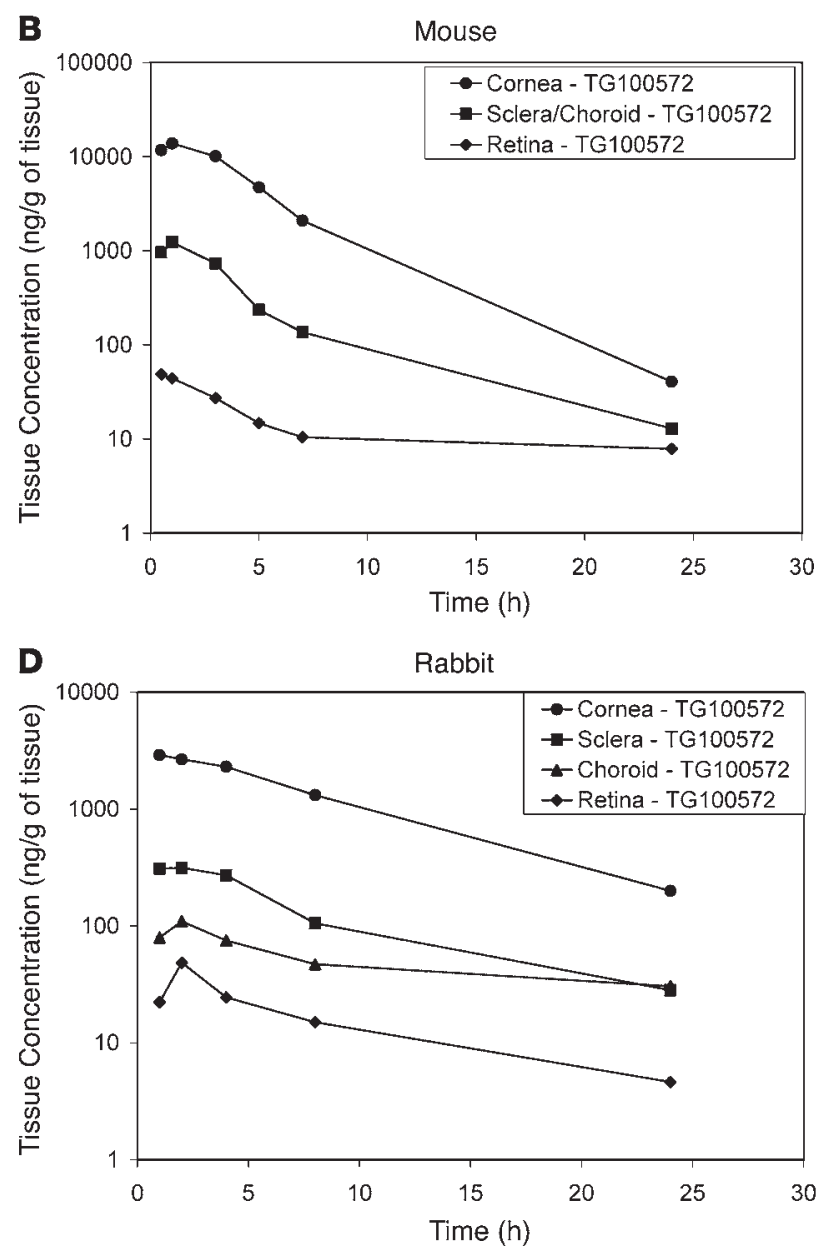

Figure 6

Topical instillation of 1.0\% TG100801 achieves posterior ocular tissue exposure to the prodrug (TG100801) and the active compound (TG100572) in mice and rabbits. Ocular tissue concentration-time profile of (A) TG100801 in mice, (B) TG100572 in mice, (C) TG100801 in rabbits, and (D) TG100572 in rabbits. 
A

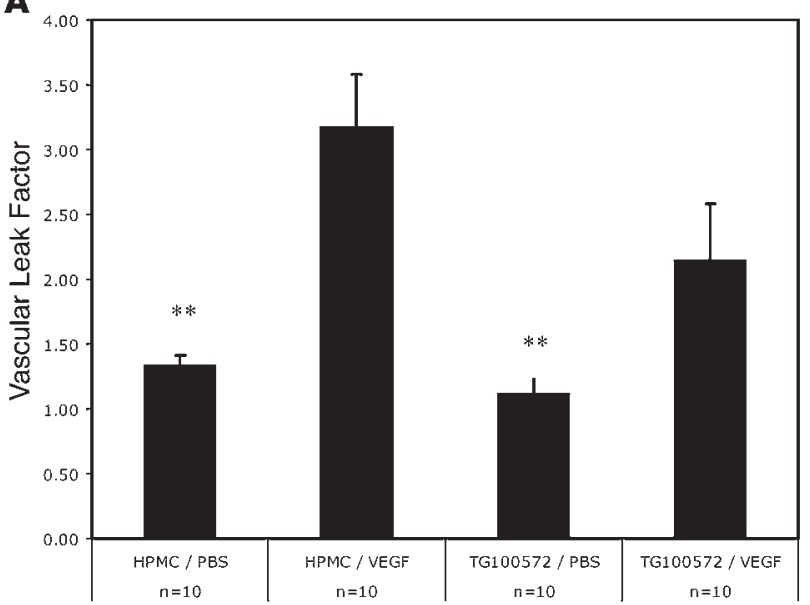

C

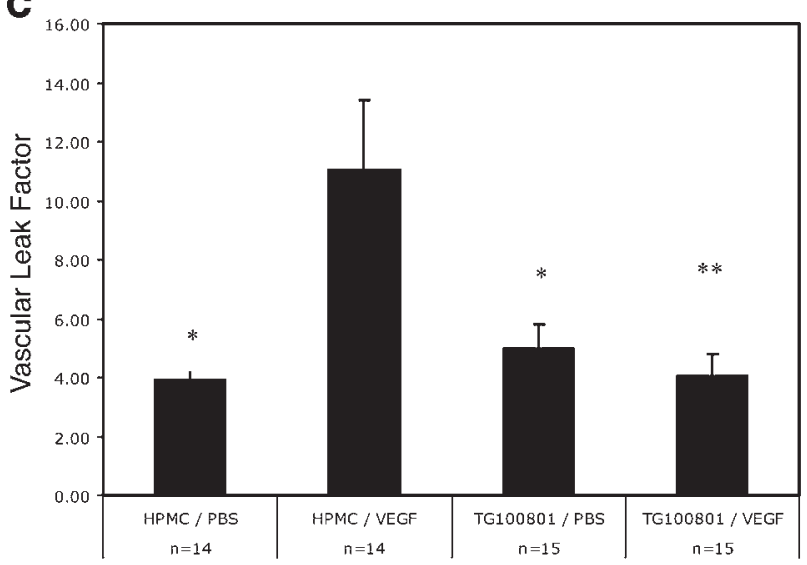

B

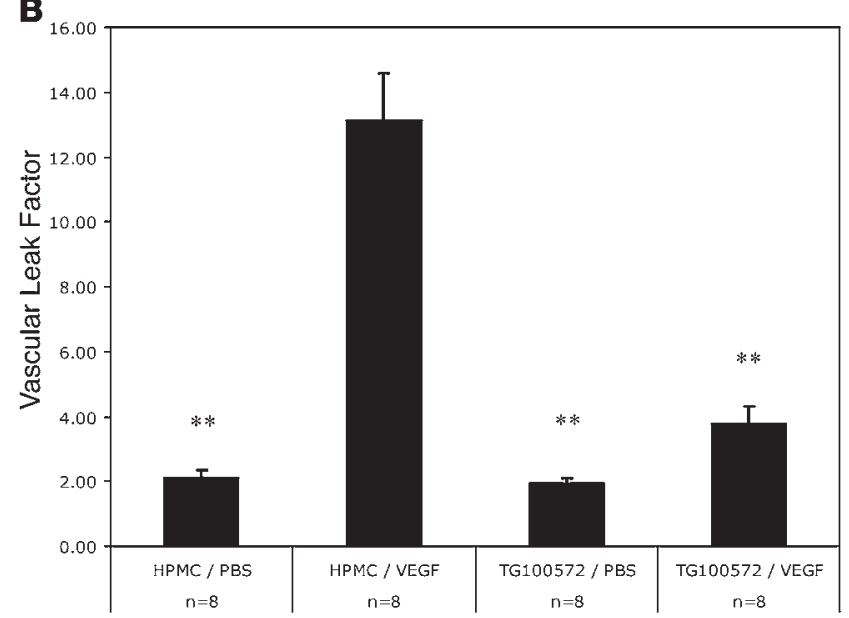

Figure 7

Topical administration of $0.7 \%$ TG100572 or $1.22 \%$ TG100801 inhibits blood-retinal barrier breakdown. EB-based quantification of retinal vascular permeability demonstrates that (A) single topical administration of TG100572 does not significantly inhibit VEGF-induced leak $(n=10)$. (B) Three topical administrations of TG100572 every 2 hours while EB circulates significantly inhibit VEGF-induced leak $(n=8)$. (C) Single topical application of TG100801 eliminates VEGF-induced leak $(n=14)$. Error bars indicate SEM. ${ }^{*} P<0.05 ;{ }^{*} P<0.01$.

In several tissues, ischemia promotes synthesis of VEGF, which then initiates leakage of fluid, macromolecules, and cells from blood vessels, promoting tissue edema (31). In the eye, VEGF administration or overexpression appears to mediate the hyperpermeability noted in several experimental models $(5,7,32)$. VEGF upregulation is also observed in several human ischemic retinopathies, such as retinal vein occlusion and diabetic retinopathy, and is believed to directly contribute to the vascular leakage and tissue edema associated with visual dysfunction $(1,3,33)$.

Src kinases are a family of cytoplasmic tyrosine kinases that participate in cellular activities through phosphorylation of intracellular substrates (34). Src and Yes have been implicated in mediating VEGF-induced alterations in vascular permeability associated with leak $(11,35)$. Mice lacking Src or Yes show no vascular leak in response to VEGF stimulation, and targeting either of these 2 SFKs is sufficient to block VEGF-induced edema in a variety of models. Another SFK, Fyn, has not been shown to play a role in VEGF-induced vascular permeability, and mice lacking Fyn show vascular leak in response to VEGF stimulation (10).

Because they are mobilized by receptors for VEGF and other stimulators of permeability (16) and in turn interact with mul- tiple intracellular signaling systems, SFKs are functionally positioned to coordinate paracellular and transcellular passage of solutes across endothelia. For example, Src kinase activation leads to phosphorylation of junctional proteins VE-cadherin, occludin, and $\beta$-catenin (36), with subsequent relaxation of intercellular contacts that normally limit leakage. Focal cell-cell and cell-extracellular matrix adhesions are also regulated by Src-dependent activation of FAK and of $\alpha v \beta 5$ integrin-mediated matrix interactions with the endothelial cell cytoskeleton (37). Cytoskeletal reorganization that disrupts interendothelial adhesions is further stimulated by Src-stimulated phosphorylation of myosin light chain (38). Formation of endothelial caveolae and fenestrae, both of which are induced by VEGF and facilitate transendothelial transport (39), also requires Src kinase activity (40).

In experimental models of stroke and myocardial infarction, local upregulation of VEGF increases vascular permeability and contributes to functional loss $(12,13)$. Src kinase inhibition reduces the edema that follows intracerebral and intravascular VEGF administration and experimental coronary and middle cerebral artery occlusions. It also prevents VEGF-induced morphological specializations affecting permeability, including fenestrae, caveo- 


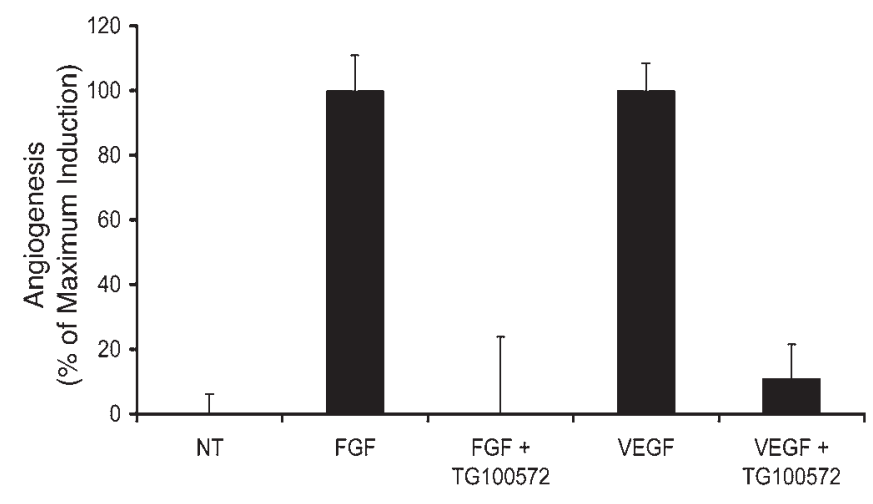

Figure 8

TG100572 hydrochloride inhibits angiogenesis induced by FGF or VEGF in vivo. The effect of TG100572 hydrochloride on angiogenesis was analyzed as described in the text. Results were normalized to the maximal angiogenic induction by either FGF or VEGF as shown on the $y$ axis. NT, no treatment. Error bars indicate SEM.

lae, leukocyte extravasation, and junctional protein modifications. Src kinase inhibition further ameliorates infarct size and neurologic deficits consequent to cardiac and cerebral vascular occlusion, respectively $(12,13)$.

We have shown that Src and Yes, both of which are expressed on vascular endothelial cells, play a role in regulating vascular permeability since exogenous VEGF failed to augment permeability in the retina of Src- and Yes-knockout mice. These results parallel those obtained in the heart and in the brain, where, in addition, a role for Fyn was excluded (12). Furthermore, we have demonstrated that pharmacological inhibition of Src kinase activity in vivo reduces both VEGFand ischemia-induced retinal vascular hyperpermeability. Thus, our data support the conclusion that Src kinases are critical mediators of VEGF- and ischemia-induced retinal vascular leakage.

Our observation that VEGF does not induce vascular exudation in $\mathrm{Src}^{-/-}$and $\mathrm{Yes}^{-/-}$mice and that this exudation is significantly reduced or completely inhibited in wild-type mice treated with Src kinase inhibitors supports the concept that Src kinase participates in multiple intracellular pathways of vascular permeability. As we have recently shown for combination angiostatic therapy, combinations of drugs that target several points in a particular cellular pathway may be more clinically efficacious (41). This may also be true for inhibiting vascular permeability changes, which could explain why a dual-acting VEGFR2/Src kinase inhibitor such as TG100572 is highly effective at preventing VEGF-mediated vascular permeability.

In contrast to the near complete dependence of VEGF-induced vascular leak on Src kinase activity, leakage due to laser treatment was only partially reversed by Src kinase inhibitors. Neither PP1, with an $\mathrm{Src} \mathrm{IC}_{50}$ of $170 \mathrm{nM}$, nor SKI-606, with a Src IC 50 of $1.2 \mathrm{nM}$, was able to completely block the laser-induced vascular leakage, but $\mathrm{Src}^{-1-}$ mice were essentially resistant to laser-induced retinal vascular leak. The $\mathrm{Yes}^{-/}$mice showed reduced leak compared with their laser-treated wild-type littermates, but not complete inhibition. This could be due to differences in the mouse background strains and their response to growth factor signaling (42) or it could be that Src kinase is more important for laser-induced vascular permeability, in which case the inhibitors might need to be delivered at a higher dose to be effective. While laser-induced vascular leakage arising from ischemia and upregulation of VEGF likely requires Src activity $(10,31)$, additional mechanisms of laserinduced permeability (e.g., local inflammatory response, direct trauma, and disruption of the outer blood-retinal barrier adjacent to the optic nerve) may be in part Src independent $(31,43)$. It is possible that following any complex pathological insult or disease process in the retina, complete blockage of exudation may not be achievable by Src kinase inhibition alone.

Permeability, strictly defined as an intrinsic property of the vascular wall, was not measured in this study; rather, we demonstrated retinal accumulation of macromolecules normally confined to the intravascular space. Extravascular deposition of intravascular contents reflects blood flow dynamics and other factors, in addition to permeability. Both retinal vasodilation and capillary nonperfusion may follow VEGF administration, for example $(44,45)$, and these exert opposing influences on blood volume and exchange surface area and thus on extravasation, independent of permeability. Nonetheless, the quantitative data in the present study are likely to reflect actual vascular permeability for several reasons. First, in preliminary experiments, results were similar irrespective of whether subjects were perfused to eliminate intravascular tracer (which varies with vasomotor and vasoocclusive effects) or whether retinal dye concentrations were calculated relative to those in nonocular vascular beds (which are presumably unaffected by intraocular hemodynamic changes due to VEGF). Second, perivascular hyperfluorescence in retinal whole-mount angiography confirmed that tracer leaked from blood vessel walls. Third, Src kinase inhibition may reduce VEGF-induced thrombosis and capillary occlusion (13), an effect that would, if anything, augment flow-dependent leakage. Fourth, tracer accumulated in the retina after laser occlusion despite angiographically documented hypoperfusion; under ischemic conditions, extravasation would decrease unless permeability increased. Finally, even though dye extravasation may not parallel retinal vascular permeability precisely, it is relevant to retinal diseases in which extravascular accumulation of fluid and macromolecules reduces vision $(1,2,25,31,46)$.

The availability of a topically applied inhibitor of vascular permeability would represent a therapeutic advance with potential widespread application in the treatment of many retinal vascular diseases, including diabetic macular edema, early choroidal neovascularization, and associated macular edema in macular degeneration and retinal vein occlusions. These data suggest that topically applied TG100801 may have clinical utility based on its ability to suppress retinal vascular leakage by inhibiting the Src and VEGFR families of tyrosine kinases.

\section{Methods}

Retinal vascular permeability. Rodent experiments were conducted with $\mathrm{BALB} / \mathrm{cByJ}$ mice, treated in accordance with approved animal subjects protocols. All animal studies were approved by the The Scripps Research Institute Animal Care and Use Committee and performed in accordance with the NIH Guide for the Care and Use of Laboratory Animals. Vascular leakage was visualized using confocal microscopy and fluorescein-dextran angiography or albumin immunohistochemistry in retinal whole mounts; in some cases, angiography was combined with isolectin immunofluorescence (47).

Retinal vascular leakage was quantified using modifications of published techniques $(5,44,48)$. Under light anesthesia (16 mg ketamine and $3 \mathrm{mg}$ xylazine, i.p.), recombinant human VEGF $(100 \mathrm{ng} / 0.5 \mu \mathrm{l})$ was injected into the vitreous cavity of one eye, and an equal volume of vehicle (PBS with 
$0.1 \% \mathrm{BSA})$ injected into the other eye. The tracer EB $(20 \mathrm{mg} / \mathrm{ml}, 0.15 \mathrm{ml}$; Sigma-Aldrich, sonicated and filtered) or FITC-conjugated albumin or dex$\operatorname{tran}(50 \mathrm{mg} / \mathrm{ml}, 0.1 \mathrm{ml}, 70 \mathrm{~S}$; Sigma-Aldrich) was then injected into the tail vein, and animals were sacrificed by anesthetic overdose $4-5$ hours later. A small amount of blood was collected via intracardiac puncture. The eyes were removed and the retinas dissected from the eyewall and optic nerve, allowed to dry, and weighed.

EB dye conjugated to serum albumin in retinas was extracted by solubilization in formamide $\left(0.2 \mathrm{ml}\right.$ per retina) at $78^{\circ} \mathrm{C}$ overnight. The resulting suspensions were placed in an ultracentrifuge at $4^{\circ} \mathrm{C}$ at $128,000 \mathrm{~g}$ for 45 minutes. EB dye in the supernatant was detected spectrophotometrically by absorbance at $620 \mathrm{~nm}$ (blue signal) and $740 \mathrm{~nm}$ (background subtracted) and measured in comparison with a standard curve. Blood samples were treated similarly but without solubilization and with centrifugation for 15 minutes at $3,550 \mathrm{~g}$ at $25^{\circ} \mathrm{C}$ and at $1: 1000$ dilution prior to spectrometric evaluation (44). EB leakage was assessed by measuring intraretinal dye accumulation using the following equation: [retinal EB concentration $(\mathrm{mg} / \mathrm{ml}) /$ retinal weight $(\mathrm{mg})] /[$ blood EB concentration $(\mathrm{mg} / \mathrm{ml}) \times$ circulation time $(\mathrm{h})]$. In experiments evaluating consequences of Src kinase inhibitors and Src mutations on VEGF-induced or laserinduced leakage (see below), blood-retinal barrier disruption was indicated as a ratio of EB dye leakage in treated vs. contralateral control eyes; i.e., as an " $n$-fold" outcome of treatment.

In vivo model of retinal hyperpermeability. An in vivo model of retinal ischemia was established as follows: retinal vessels in 1 eye were occluded using a green wavelength diode laser (power $200 \mathrm{~mW}$, spot size $50 \mu \mathrm{m}$, duration 0.05 second, repeat mode with 20-30 applications), centered on the major veins as they branched off the vascular trunk within the optic nerve head. The end point of treatment was cessation of blood flow in veins, though adjacent arteries often exhibited partial blanching. Placement of laser burns adjacent to the optic nerve head avoided retinal damage that complicates interpretation of dye extravasation and makes later dissection difficult (49). EB dye was administered 3 days after laser treatment and permeability then determined as described above.

General considerations in experimental design for delivery of VEGF and in hibitors. There were 2 broad issues to consider when designing the experiments. One was controlling for VEGF-induced leak when using EB dye to measure changes in vascular permeability. The second was the administration of the Src kinase antagonist and limitation of possible crossover from one eye to the other in instances where we would have liked to do paired eye experiments (e.g., each eye receiving distinct compounds such as control vehicle vs. antagonist) in topical administration.

With regard to controlling for VEGF-induced leak, it was important to have an internal PBS control in each animal to which we could compare the leak induced by VEGF. This internal control also accounted for any small differences in the quantity of EB that was systemically injected from animal to animal. By comparing left (PBS) to right (VEGF) eye, each of which had been exposed to the same amount of tracer (EB), we felt confident that differences in leak observed were actually due to VEGF rather than differences in quantities of injected tracer. Furthermore, this experimental design gave us confidence that the VEGF used in each experiment was active and able to induce vascular permeability as expected, since in the groups treated with vehicle we were able to see reproducible VEGF-induced leak when compared with the contralateral internal PBS control-injected eye.

The SFK kinase antagonists, PP1 and SKI-606, were both administered systemically, so by default, both eyes were exposed to either SFK antagonist or vehicle control. In the case of the topically administered Src kinase antagonists, a fraction of any eye drop applied would diffuse into the lacrimal ducts, which connect to the nasopharynx, a highly vascularized region. Should this have happened, there was the possibility that a small amount of the drug would enter systemic circulation. This could confound the results if we had applied vehicle, for example, to the left eye and antagonist to the right eye, with both eyes receiving either PBS injections or VEGF injections. Furthermore, because we were dealing with mice, there was the concern that instinctual grooming and pawing by the mice could cause cross-contamination if contralateral eyes had not received identical topical treatment.

The number of mice in most groups was in the range of $10-12$, with a minimum group size of 7 and a maximum group size of 36 . In all cases, statistical significance was determined by 2 -sample $t$ test assuming equal variance. Error bars depict SEM.

Systemic Src kinase inhibition. Pharmacological SFK inhibition was achieved using SFK inhibitors 4-amino-5-(4-methylphenyl)-7-(t-butyl) pyrazolo[3,4dipyrimidine (PP1) (1.5 mg/kg i.p.; BIOMOL Research) or SKI-606 (5 mg/kg i.v.; Wyeth Pharmaceuticals) (19). PP1 was dissolved in DMSO and further diluted in PBS. SKI-606 was dissolved in 10\% ethanol and 50\% PEG-400 (Sigma-Aldrich). A third experiment tested the efficacy of Avastin vs. PBS vehicle (systemic delivery) to compare the efficacy of SFK inhibitors with an FDA-approved, commercially available product. The SFK inhibitors were injected 15 minutes prior to VEGF administration or, when using the in vivo laser model, 15 minutes prior to EB administration.

Assessment of retinal vascular permeability in SFK-knockout mice. To further clarify the specificity of SFK activity in retinal vascular leakage, we employed mice lacking SFK known to be expressed on vascular endothelial cells, specifically, $\mathrm{Src}^{-/-}$and $\mathrm{Yes}^{-/-}$mice $(11,35)$. Before their inclusion in experimental procedures, offspring of $\mathrm{Src}^{+/-}$or $\mathrm{Yes}^{+/-}$parents (obtained from The Jackson Laboratory and as a generous gift from Phil Soriano, Fred Hutchinson Cancer Research Center, Seattle, Washington, USA) were genotyped, and their genotypic status was masked during performance of subsequent interventions. Homozygous knockouts $\left(\mathrm{Src}^{-/}\right.$, $\mathrm{Yes}^{-/-}$) were compared with respective homozygous wild-type littermates $\left(\mathrm{Src}^{+/+}, \mathrm{Yes}^{+/+}\right) . \mathrm{Yes}^{-/-}$mice appeared grossly normal; $\mathrm{Src}^{-/-}$mice exhibited reduced body weight and dental anomalies $(35,50)$.

Topical SFK inhibition of VEGF-induced retinal vascular permeability. A topically applied SFK inhibitor and its prodrug were tested for their ability to block VEGF-induced vascular extravasation. The active compound, TG100572, is an ATP-competitive multitargeted tyrosine kinase inhibitor, whereas the prodrug, TG100801 is devoid of kinase inhibitory activity. It is cleaved into the active form (TG100572) in ocular tissues. In one experiment, TG100572 (10 $\mu$ l eyedrop, $7 \mathrm{mg} / \mathrm{ml}$; TargeGen) was compared with its liposomal vehicle (25\% Phospholipon 90G) either delivered in a single application or applied every 2 hours over the course of a 6-hour experiment. In another experiment, its prodrug TG100801 (10 $\mu \mathrm{l}$ eyedrop, $10 \mathrm{mg} / \mathrm{ml}$; TargeGen) was compared with liposomal vehicle (25\% Phospholipon 90G) delivered in a single application.

Quantification and pharmacokinetics of TG100572 and TG100801 in ocular tissue and plasma. One $10 \mu \mathrm{l}$ drop of the topical drug being tested $(1.2 \%$ TG100801, $n=54$ mice) was applied to each eye of adult male mice. Animals were sacrificed at $0.5,1,3,5,7$, and 24 hours after instillation $(n=9$ mice per time point). Eyes were enucleated and dissected to obtain the cornea, retina, and eye cup (sclera and choroid). Plasma samples were also obtained at the time of euthanasia.

The ocular tissue concentrations were also assessed in rabbits following topical bilateral instillation of $40 \mu \mathrm{l}$ of $1.0 \%$ TG100801. Male Dutch-belted rabbits $(n=15)$ were sacrificed at 1, 2, 4, 8, and 24 hours after instillation ( $n=3$ rabbits per time point). Plasma samples were isolated, and eyes were enucleated and dissected in cornea, sclera, choroids, and retina.

For each time point, individual eye tissue samples were weighed and homogenized in $0.5 \mathrm{ml}$ of RIPA buffer using FP120 FastPrep (ThermoSavant) and extracted with $0.5 \mathrm{ml}$ of acetonitrile (containing internal standard). The supernatants $(800 \mu \mathrm{l})$ were dried and reconstituted into $400 \mu \mathrm{l}$ of 8:2 DMSO/ 
water for analysis. Plasma samples were extracted by addition of a 2-fold excess of acetonitrile containing internal standard followed by centrifugation. The supernatants were isolated for analysis. TG100572 or TG100801 levels were quantified from processed plasma and ocular tissue samples by liquid chromatography/mass spectrometry-mass spectrometry and compared with controls (processed plasma and ocular tissue from naive mice or rabbits).

Assessment of antiangiogenic activity of TG100572 and TG100801 in the mouse Matrigel angiogenesis assay. The mouse Matrigel angiogenesis assay was used to examine the angiostatic activity of TG100572 hydrochloride essentially as previously described. In brief, 5 immunocompromised (nu/nu) mice per group were implanted in the flank with $400 \mu \mathrm{l}$ of growth factor-depleted Matrigel (BD) infused with either $20 \mathrm{ng} / \mathrm{ml}$ VEGF or FGF (R\&D Systems) as indicated. Four hours after implantation, the angiostatic activity of TG100572 hydrochloride was evaluated by administering TG100572 hydrochloride at $5 \mathrm{mg} / \mathrm{kg}$ i.p. for 5 days. On day 5 , the mice were i.v. injected with $50 \mu \mathrm{g}$ of biotin-labeled endothelial-binding lectin Griffonia (Bandeiraea) simplicifolia I, isolectin B4 (Vector Laboratories). Four hours after the final dose, the Matrigel plugs were resected and dissociated by grinding the plugs in RIPA buffer ( $50 \mathrm{mM}$ Tris- $\mathrm{HCl}$, $\mathrm{pH} 7.4$; 1\% NP-40; $0.25 \%$ Na-deoxycholate; 150 mM NaCl; 1 mM EDTA; 1 mM phenylmethylsulfonyl fluoride; $1 \mu \mathrm{g} / \mathrm{ml}$ aprotinin; $1 \mu \mathrm{g} / \mathrm{ml}$ leupeptin; $1 \mu \mathrm{g} / \mathrm{ml}$ pepstatin; $1 \mathrm{mM} \mathrm{Na}_{3} \mathrm{VO}_{4}$; and $1 \mathrm{mM} \mathrm{NaF}$ ). The biotin content of each plug was quantified by ELISA analysis using React-Bind streptavidin-coated plates (Pierce Biotechnology) to capture the biotin endothelial cell marker and anti-biotin antibodies conjugated to peroxidase (Vector
Laboratories) as a signal. The peroxidase content per well was determined by addition of 3,3',5,5'-tetramethylbenzidine dihydrochloride (TMB) substrate, which undergoes a colorimetric change in the presence of peroxidase. Color-shifted TMB was quantified by measurement of the $650-\mathrm{nm}$ absorbance on a SpectraMax Plus microplate reader (Molecular Devices). Angiogenesis is reported as a percentage of the induction observed in the growth factor-positive control, which did not receive inhibitor treatment. Data are presented as mean $\pm \mathrm{SD}$. Statistics were derived using ANOVA analysis by Microsoft Excel (Microsoft Corp.).

\section{Acknowledgments}

This work was supported by grants to M. Friedlander from the NIH (National Eye Institute EY11254), the MacTel Foundation, the Scripps Mericos/Fonseca Fund, and the V. Kann Rassmussen Foundation. R.F. Gariano was supported by the Skaggs Clinical Scholars Program. D.A. Cheresh received grant support from the NIH (National Eye Institute EY 014174).

Received for publication July 23, 2007, and accepted in revised form April 2, 2008.

Address correspondence to: Martin Friedlander, Department of Cell Biology, MB28, The Scripps Research Institute, 10550 N. Torrey Pines Rd., La Jolla, California 92037, USA. Phone: (858) 784-9138; Fax: (858) 783-9135; E-mail: friedlan@scripps.edu.
1. Cunha-Vaz, J.G. 1983. Studies on the pathophysiology of diabetic retinopathy. The blood-retinal barrier in diabetes. Diabetes. 32(Suppl. 2):20-27.

2. Chahal, P.S., Fallon, T.J., and Kohner, E.M. 1986. Measurement of blood-retinal barrier function in central retinal vein occlusion. Arch. Ophthalmol. 104:554-557.

3. Caldwell, R.B., et al. 2003. Vascular endothelial growth factor and diabetic retinopathy: pathophysiological mechanisms and treatment perspectives. Diabetes Metab. Res. Rev. 19:442-455.

4. Boyd, S.R., et al. 2002. Correlation of increased vascular endothelial growth factor with neovascularization and permeability in ischemic central vein occlusion. Arch. Ophthalmol. 120:1644-1650.

5. Qaum, T., et al. 2001. VEGF-initiated blood-retinal barrier breakdown in early diabetes. Invest. Ophthalmol. Vis. Sci. 42:2408-2413.

6. Antonetti, D.A., et al. 1998. Vascular permeability in experimental diabetes is associated with reduced endothelial occludin content: vascular endothelial growth factor decreases occludin in retinal endothelial cells. Penn State Retina Research Group. Diabetes. 47:1953-1959.

7. Vinores, S.A., et al. 1997. Upregulation of vascular endothelial growth factor in ischemic and nonischemic human and experimental retinal disease. Histol. Histopathol. 12:99-109.

8. Fine, H.F., Baffi, J., Reed, G.F., Csaky, K.G., and Nussenblatt, R.B. 2001. Aqueous humor and plasma vascular endothelial growth factor in uveitis-associated cystoid macular edema. Am. J. Ophthalmol. 132:794-796.

9. Rosenfeld, P.J., et al. 2006. Ranibizumab for neovascular age-related macular degeneration. $N$. Engl. J. Med. 355:1419-1431.

10. Eliceiri, B.P., et al. 1999. Selective requirement for $S R C$ kinases during VEGF-induced angiogenesis and vascular permeability. Mol. Cell. 4:915-924.

11. Yuan, S.Y. 2002. Protein kinase signaling in the modulation of microvascular permeability. Vascul. Pharmacol. 39:213-223.

12. Paul, R., et al. 2001. SRC deficiency or blockade of $S R C$ activity in mice provides cerebral protection following stroke. Nat. Med. 7:222-227.

13. Weis, S., et al. 2004. SRC blockade stabilizes a Flk/ cadherin complex, reducing edema and tissue injury following myocardial infarction. J. Clin. Invest. 113:885-894.

14. Owens, D.W., et al. 2000. The catalytic activity of the $S R C$ family kinases is required to disrupt cadherin-dependent cell-cell contacts. Mol. Biol. Cell. 11:51-64.

15. Kevil, C.G., Payne, D.K., Mire, E., and Alexander, J.S. 1998. Vascular permeability factor/vascular endothelial cell growth factor-mediated permeability occurs through disorganization of endothelial junctional proteins. J. Biol. Chem. 273:15099-15103.

16. Weis, S.M., and Cheresh, D.A. 2005. Pathophysiological consequences of VEGF-induced vascular permeability. Nature. 437:497-504.

17. Chou, M.T., Wang, J., and Fujita, D.J. 2002. SRC kinase becomes preferentially associated with the VEGFR, KDR/Flk-1, following VEGF stimulation of vascular endothelial cells. BMC Biochem. 3:32.

18. Weis, S., Cui, J., Barnes, L., and Cheresh, D. 2004. Endothelial barrier disruption by VEGF-mediated $S R C$ activity potentiates tumor cell extravasation and metastasis. J. Cell Biol. 167:223-229.

19. Boschelli, D.H., et al. 2001. Optimization of 4phenylamino-3-quinolinecarbonitriles as potent inhibitors of SRC kinase activity. J. Med. Chem. 44:3965-3977.

20. Blake, R.A., et al. 2000. SU6656, a selective SRC family kinase inhibitor, used to probe growth factor signaling. Mol. Cell. Biol. 20:9018-9027.

21. Golas, J.M., et al. 2003. SKI-606, a 4-anilino-3-quinolinecarbonitrile dual inhibitor of SRC and $\mathrm{Abl}$ kinases, is a potent antiproliferative agent against chronic myelogenous leukemia cells in culture and causes regression of K562 xenografts in nude mice. Cancer Res. 63:375-381.

22. Nunes, I., Higgins, R.D., Zanetta, L., Shamamian, P., and Goff, S.P. 2001. c-abl is required for the development of hyperoxia-induced retinopathy. J. Exp. Med. 193:1383-1391.

23. Werdich, X.Q., and Penn, J.S. 2006. Specific involve- ment of SRC family kinase activation in the pathogenesis of retinal neovascularization. Invest. Ophthalmol. Vis. Sci. 47:5047-5056.

24. Noronha, G., et al. 2007. Discovery of [7- (2,6dichlorophenyl)-5-methylbenzo $[1,2,4]$ triazin3-yl]-[4- (2-pyrrolidin-1-ylethoxy)phenyl]amine - a potent, orally active $S R C$ kinase inhibitor with anti-tumor activity in preclinical assays. Bioorg. Med. Chem. Lett. 17:602-608.

25. Ciulla, T.A., Amador, A.G., and Zinman, B. 2003. Diabetic retinopathy and diabetic macular edema: pathophysiology, screening, and novel therapies. Diabetes Care. 26:2653-2664.

26. Ferris, F. 1996. Early photocoagulation in patients with either type I or type II diabetes. Trans. Am. Ophthalmol. Soc. 94:505-537.

27. Avery, R.L., et al. 2006. Intravitreal bevacizumab (Avastin) for neovascular age-related macular degeneration. Ophthalmology. 113:363-372e5.

28. Ferrara, N., Gerber, H.P., and LeCouter, J. 2003. The biology of VEGF and its receptors. Nat. Med. 9:669-676.

29. Alon, T., et al. 1995. Vascular endothelial growth factor acts as a survival factor for newly formed retinal vessels and has implications for retinopathy of prematurity. Nat. Med. 1:1024-1028.

30. Storkebaum, E., Lambrechts, D., and Carmeliet, P. 2004. VEGF: once regarded as a specific angiogenic factor, now implicated in neuroprotection. Bioessays. 26:943-954.

31. Bates, D.O., and Harper, S.J. 2002. Regulation of vascular permeability by vascular endothelial growth factors. Vascul. Pharmacol. 39:225-237.

32. Tolentino, M.J., et al. 2002. Pathologic features of vascular endothelial growth factor-induced retinopathy in the nonhuman primate. Am. J. Ophthalmol. 133:373-385.

33. Frank, R.N. 2004. Diabetic retinopathy. N. Engl. J. Med. 350:48-58.

34. Mathews, M.K., Merges, C., McLeod, D.S., and Lutty, G.A. 1997. Vascular endothelial growth factor and vascular permeability changes in human diabetic retinopathy. Invest. Ophthalmol. Vis. Sci. 38:2729-2741. 
35. Lowell, C.A., and Soriano, P. 1996. Knockouts of $S R C$-family kinases: stiff bones, wimpy T cells, and bad memories. Genes Dev. 10:1845-1857.

36. Tinsley, J.H., Ustinova, E.E., Xu, W., and Yuan, S.Y. 2002. SRC-dependent, neutrophil-mediated vascular hyperpermeability and beta-catenin modification. Am. J. Physiol. Cell Physiol. 283:C1745-C1751.

37. Eliceiri, B.P., et al. 2002. SRC-mediated coupling of focal adhesion kinase to integrin alpha (v)beta5 in vascular endothelial growth factor signaling. $J$. Cell Biol. 157:149-160.

38. Birukov, K.G., et al. 2001. Differential regulation of alternatively spliced endothelial cell myosin light chain kinase isoforms by p60 (SRC). J. Biol. Chem. 276:8567-8573.

39. Hofman, P., et al. 2000. VEGF-A induced hyperpermeability of blood-retinal barrier endothelium in vivo is predominantly associated with pinocytotic vesicular transport and not with formation of fenestrations. Vascular endothelial growth factor-A. Curr. Eye Res. 21:637-645.
40. Sanguinetti, A.R., Cao, H., and Corley Mastick, C. 2003. Fyn is required for oxidative- and hyperosmotic-stress-induced tyrosine phosphorylation of caveolin-1. Biochem. J. 376:159-168.

41. Dorrell, M.I., Aguilar, E., Scheppke, L., Barnett, F.H., and Friedlander, M. 2007. Combination angiostatic therapy completely inhibits ocular and tumor angiogenesis. Proc. Natl. Acad. Sci. U. S. A. 104:967-972.

42. Rogers, M.S., and D'Amato, R.J. 2006. The effect of genetic diversity on angiogenesis. Exp. Cell Res. 312:561-574.

43. Lindbom, L. 2003. Regulation of vascular permeability by neutrophils in acute inflammation. Chem. Immunol. Allergy. 83:146-166.

44. Xu, Q., Qaum, T., and Adamis, A.P. 2001. Sensitive blood-retinal barrier breakdown quantitation using Evans blue. Invest. Ophthalmol. Vis. Sci. 42:789-794.

45. He, H., et al. 1999. Vascular endothelial growth factor signals endothelial cell production of nitric oxide and prostacyclin through flk-1/KDR activation of c-SRC. J. Biol. Chem. 274:25130-25135.

46. Watzke, R.C., et al. 2000. A comparison of stereoscopic fluorescein angiography with indocyanine green videoangiography in age-related macular degeneration. Ophthalmology. 107:1601-1606.

47. Dorrell, M.I., Aguilar, E., and Friedlander, M 2002. Retinal vascular development is mediated by endothelial filopodia, a preexisting astrocytic template and specific R-cadherin adhesion. Invest. Ophthalmol. Vis. Sci. 43:3500-3510.

48. Derevjanik, N.L., et al. 2002. Quantitative assessment of the integrity of the blood-retinal barrier in mice. Invest. Ophthalmol. Vis. Sci. 43:2462-2467.

49. Miller, J.W., et al. 1994. Vascular endothelial growth factor/vascular permeability factor is temporally and spatially correlated with ocular angiogenesis in a primate model. Am. J. Pathol. 145:574-584.

50. Stein, P.L., Vogel, H., and Soriano, P. 1994. Combined deficiencies of SRC, Fyn, and Yes tyrosine kinases in mutant mice. Genes Dev. 8:1999-2007. 